\title{
ГЕНЕТИЧЕСКИЙ АЛГОРИТМ ДЛЯ ЗАДАЧИ ОДНОМЕРНОЙ УПАКОВКИ ОБЪЕКТОВ
}

\section{GENETIC ALGORITHM FOR THE PROBLEM OF ONE-DIMENSIONAL PACKING OF OBJECTS}

\section{A. Dvoryankin I. Ibrahim}

Summary. Genetic algorithms (GAs) are adaptive search algorithms inspired by the process of natural selection, which are useful for optimization purposes. These methods do not ensure exact optimal solution; however, they give good approximated sets of solutions in a short time compared to other kind of algorithms, which takes forever to solve a given problem. The genetic algorithms are useful for solving mostly NP-hard problems, especially the bin-packing problem. In this article, we used genetic algorithm to tackle the placement of loaded segment problem to find permutation of segments with minimum deviation from a given center of gravity. We should also note that this approach does not ensure exact optimal solution but we are sure to have an approximate solution and also it will serve as basis for comparisons with other approximate algorithms in the future.

Keywords: one-dimensional packing, force, loaded segments, genetic algorithm, chromosome, mutation, crossover, center of gravity.

\author{
Дворянкин Александр Михайлович \\ Д.т.н., профессор, Волгоградский государственный \\ технический университет, Волгоград \\ dvam@vstu.ru \\ Ибрагим Исмаил Адаму \\ Волгоградский государственный технический \\ университет, Волгоград \\ ismometrical@gmail.com
}

Аннотация. Генетические алгоритмы (ГА) - это алгоритмы адаптивного поиска, основанные на принципе естественного отбора, которые полезны для целей оптимизации. Эти методы не обеспечивают точного оптимального решения, однако дают хорошие приближенные наборы решений за короткое время по сравнению с точными алгоритмами, которые требуют практически бесконечного времени для решения той же задачи. Генетические алгоритмы полезны для решения в основном NP-полных задач, в частности, проблемы упаковки в контейнеры. В этой статье мы использовали генетический алгоритм для решения проблемы размещения нагруженных отрезков, чтобы найти перестановку отрезков с минимальным отклонением от заданного центра тяжести. Следует отметить, что такой подход не гарантирует точного оптимального решения, но обеспечивает приблизительное решение с некоторой точностью, что будет служить основой для сравнения эффективности приближенных алгоритмов в будущем.

Ключевые слова: одномерная упаковка, сила, нагруженные отрезки, генетический алгоритм, хромосома, мутация, кроссинговер, центр тяжести.

\section{Введение}

И нтерес к различным постановкам и алгоритмам решения задач размещения и, в частности, к задаче оптимальной одномерной упаковки или «задаче о ранце» [1,2] не ослабевает [3,4]. Задача одномерной упаковки является задачей комбинаторной оптимизации и относится к классу NP-полных [6]. Особенность рассматриваемой постановки задачи размещения нагруженных отрезков состоит в том, чтобы достичь положения центра тяжести «ранца» как можно ближе к заданному. В статье рассматривается модель и задача размещения отрезков, нагруженных сосредоточенной силой [7]. Критерий оптимального размеще-

ния - минимальное отклонение центра тяжести от заданного значения.

Эта задача возникает при проектировании компоновки самолета, размещения груза на транспортном средстве, для которого положение центра тяжести является важным. Предложен генетический алгоритм для решения задачи одномерной упаковки объектов.

\section{Mosenb}

Будем использовать обозначения отрезков, понятие соединения отрезков и формулы вычисления центра тяжести соединения отрезков, предложенные в работе [7]. 
Пусть $A=(a, p, b)$ - это горизонтальный отрезок, где $p$ - это точка приложения к нему вертикальной силы (вес), $a$ - расстояние от левого конца отрезка до $p, b-$ расстояние от $p$ до правого конца отрезка. Длина отрезка $\mathrm{d}$ определяется как $a+b$, при условии, что $a$ и $b$ строго больше нуля, а вес $p$ неотрицателен. Примем обозначение различных отрезков нижними индексами, как отрезка в целом, так и его компонентов: $A_{x}=\left(a_{x}, p_{x}, b_{x}\right)$ или $A_{j}=\left(a_{j}, p_{j}, b_{j}\right)$. Компоненты отрезка А будем также записывать как $a=a(A), p=p(A), b=$ $b(A)$. Соединение отрезков $A_{x}$ и $A_{y}$ примет форму $A_{x y}=$ $A_{x} A_{y}$. При этом компоненты соединения $A_{x y}=\left(a_{x y}, p_{x y}\right.$, $b_{x y}$ ) вычисляются следующим образом:

$a_{x y}=a\left(A_{x y}\right)=\left[p_{x} a_{x}+p_{y}\left(a_{x}+b_{x}+a_{y}\right)\right], p_{x y}=p\left(A_{x y}\right)=$ $p_{x}+p_{y}, b_{x y}=b\left(A_{x y}\right)=d_{x}+d_{y}-a_{x y}$.

\section{Постановка заАачи}

Пусть заданы $n$ отрезков $A_{1}, A_{2}, \ldots, A_{n}$ и некоторое число $c>0$. Для любой перестановки $w=\left(i_{1}, i_{2}, \ldots, i_{n}\right) \in J_{n}$ сопоставим соединение отрезков: $A_{w}=A_{i_{1}} A_{i_{2}} \ldots A_{i_{n}}$. Функция $a\left(A_{w}\right)$ является функцией от перестановки $w$.

Для заданного множества $n$ отрезков и числа $c$ определим функцию $\delta$ :

$$
\begin{aligned}
& \delta\left(i_{1}, i_{2}, \ldots, i_{n}\right)=\left|a\left(A_{i_{1}} A_{i_{2}} \ldots A_{i_{n}}\right)-c\right|, \\
& \text { где }\left(i_{1}, i_{2}, \ldots, i_{n}\right) \ni J_{n} .
\end{aligned}
$$

Задача упаковки: найти перестановку $\left(i_{1}^{0}, i_{2}^{0}, \ldots, i_{n}^{0}\right) \in J_{n}$, на которой достигается минимум функции:

$$
\begin{aligned}
& \text { д( } \left.i_{1}^{0}, i_{2}^{0}, \ldots, i_{n}^{0}\right)=\min _{\left(i_{1}, i_{2}, \ldots, i_{n}\right) \in J_{n}} д\left(i_{1}, i_{2}, \ldots, i_{n}\right) \\
& \text { Мето икка решений } \\
& \text { генетического алгоритма }
\end{aligned}
$$

Для решения поставленной задачи за основу был взят классический генетический алгоритм. Определим основные элементы генетического алгоритма в терминах решаемой задачи. Хромосомы будут представлять перестановки отрезков, ген будет представлять отрезок. Для кодирования решения использовано целочисленное кодирование: вектор целых чисел в диапазоне от 1 и до $n$, где $n-$ это количество отрезков, каждое число вектора определяет позицию отрезка в перестановке. В качестве целевой функции, которая описывает точность решения задачи, будем использовать функцию отклонения $\partial$, которая на вход получает перестановку отрезков и возвращает отклонение от желаемого центра тяжести.
Для инициализации популяции будем использовать стратегию случайной популяции, т.е. заполнение исходной популяции выполняется случайным образом. А для отбора случайным образом выбираются родители из существующей популяции; преимуществом данной стратегии является то, что она не требует дополнительных вычислений.

В качестве оператора скрещивания (кроссинговера) был выбран одноточечный кроссинговер, который моделируется следующим образом. Пусть есть две родительские особи с хромосомами. Случайным образом определяется точка внутри хромосомы (точка разрыва) р где $1 \leq p \leq n$, в которой обе хромосомы делятся на две части и обмениваются ими [8]. Оператор мутации в генетических алгоритмах используется для того, что бы «выбивать» решения из локальных оптимумов, которые далеки от глобальных. Для мутации генов используется простой способ обмена двух генов (отрезков).

\section{АлгорИтм}

Для приближенного решения задачи размещения нагруженных отрезков предлагается следующий генетический алгоритм.

1. Проинициализировать параметр генетического алгоритма k - количество перестановок в начальной популяции и g - количество поколений.

2. Случайным образом сгенерировать k перестановок отрезоков $G=\left\{P_{1}, P_{2}, \ldots, P_{k}\right\}$.

3. Для каждого поколения от 1 до g выполнять:

3.1. Вычислить значение центра тяжести каждой перестановки и с помощью этого вычислить значение отклонения для каждой перестановки в популяции.

3.2. Скопировать лучшую перестановку и ее значение отклонения. Рассчитать среднее отклонение.

3.3. Пока (количество потомков перестановки меньше k) выполнять:

3.3.1. Случайным образом выбрать две перестановки $P_{i}, P_{j}$ из популяции $G$, где $1 \leq i, j \leq k$ и $i \neq j$

3.3.2. Выполнить операцию скрещивания на основе порядка между этими двумя перестановками $\mathrm{P}_{i}$ и $\mathrm{P}_{j}$, чтобы получить новую перестановку отрезков $P_{\text {потомка }}$.

3.3.3. Случайным образом поменять местами два отрезка в перестановке $P_{\text {потомка }}$ который был получен на предыдущем шаге.

3.3.4. Увеличить счетчик в зависимости от количества потомков перестановки 1 или 2.

3.4. конец цикла Пока. 
Таблица 1. Исходные данные

\begin{tabular}{|l|l|l|l|}
\hline$A$ & $a(A)$ & $p(A)$ & $b(A)$ \\
\hline$A_{1}$ & 1 & 5 & 8 \\
\hline$A_{2}$ & 5 & 5 & 9 \\
\hline$A_{3}$ & 4 & 5 & 7 \\
\hline$A_{4}$ & 2 & 5 & 8 \\
\hline$A_{5}$ & 1 & 5 & 6 \\
\hline$A_{6}$ & 5 & 5 & 3 \\
\hline$A_{7}$ & 5 & 15 & 8 \\
\hline
\end{tabular}

Таблица 2. Результаты работы алгоритмов

\begin{tabular}{|l|l|l|}
\hline$w$ & Генетический алгоритм & Метод полного перебора \\
\hline Оптимальное положение центра тяжести & $(4,6,5,1,7,2,3)$ & $(5,4,6,3,1,2,7)$ \\
\hline$\partial$ & 35.1111 & 35 \\
\hline Время решения задачи, сек. & 0.1111 & 0.0000 \\
\hline
\end{tabular}

3.5. Построить промежуточный набор перестановок из множества потомков перестановки, которая была получена.

3.6. Создать новый набор перестановок из старого набора и вновь созданного набора потомков перестановки путем замены перестановок с худшим значением пригодности перестановками с лучшим значением приспособленности.

3.7. Проверить: если уже найдено максимально оптимальное решение - перестановка со значением отклонения, равным 0, то перейти к шагу 5.

4. конец цикла Для.

5. Решение - соответствующая перестановка $P$, с минимальной $f$.

6. Распечатать лучшую перестановку и ее значение критерия.

Пример исходных данных при $k=10, g=3, n=7$ и $c=35$ приведен в таблице 1.

При $k=3$ найдено приближенное решение: $w=(4,6,5,1,7,2,3)$,д $=0.1111$. Отклонение от глобального минимума не превышает значения $\delta$.
Применение алгоритма полного перебора дает глобальный минимум на перестановке $w=(5,4,6,3,1,2$, 7), $\delta=0.0000$.

Пример результатов работы генетического алгоритма и метода полного перебора приведен в таблице 2.

\section{Зак^Ючение}

Приведена постановка задачи размещения отрезков, нагруженных сосредоточенной силой. Критерием оптимального размещения является минимальное отклонение центра тяжести результирующего отрезка от заданного значения. Генетический алгоритм дает приближенное решение. Временная сложность алгоритма составляет $O\left(n^{4}\right)$. Алгоритм хорошо подходит для большого количества отрезков, когда стандартные методы оказываются неэффективными по сравнению с методами эволюционного моделирования. Время работы генетического алгоритма, в сравнении с алгоритмом полного перебора, уменьшилось в 5-10 раз.

\section{ЛИТЕРАТУРА}

1. Беллман Р., Дрейфус С. Прикладные задачи динамического программирования. — М.: Наука, 1965.— 460 c.

2. Mongeau, M., Be` S, C., 2003. Optimization of aircraft container loading. IEEE Transactions on Aerospace and Electronic Systems 39, 1, 140-150.

3. Залюбовский В.В. 0 представлении перестановками допустимых решений одномерной задачи упаковки в контейнеры. // Труды XIII Байкальской международной школы-семинара «Методы оптимизации». Том 1: Иркутск, ИСЭМ СО АН РАН. — 2005. с. 461-467.

4. Wim Vancroonenburg, Jannes Verstichel, Karel Tavernier, Greet Vanden Berghe. Automatic air cargo selection and weight balancing: A mixed integer programming approach. Transportation Research Part E: Logistics and Transportation Review, Volume 65, May 2014, Pages 70-83. D0l: https://doi. org/10.1016/j.tre.2013.12.013. 
5. Kaluzny, Bohdan L.; Shaw, R.H.A. David. Optimal aircraft load balancing. International Transactions in Operational Research, Volume 16, Number 6, November 2009, pp. 767-787(21). DOI: https://doi.org/10.1111/j.1475-3995.2009.00723.x

6. Гэри М., Джонсон Д. Вычислительные машины и труднорешаемые задачи. - М.: Мир, 1982. — 416 с.

7. А.М. Дворянкин, М.Б. Кульцова, И.Г. Жукова. Задача размещения нагруженных отрезков./ А.М. Дворянкин, М.Б. Кульцова, И.Г. Жукова // Известия ВолгГТУ. Сер. Актуальные проблемы управления, вычислительной техники и информатики в технических системах.— Волгоград, 2017.- № 8 (203). - C. 29-34.

8. Lozano M., Herrera F., Krasnogor N., Molina D. Real-Coded Memetic Algorithms with Crossover Hill-Climbing. Evolutionary Computation 12(3), 2004.- Pp. 273-302.

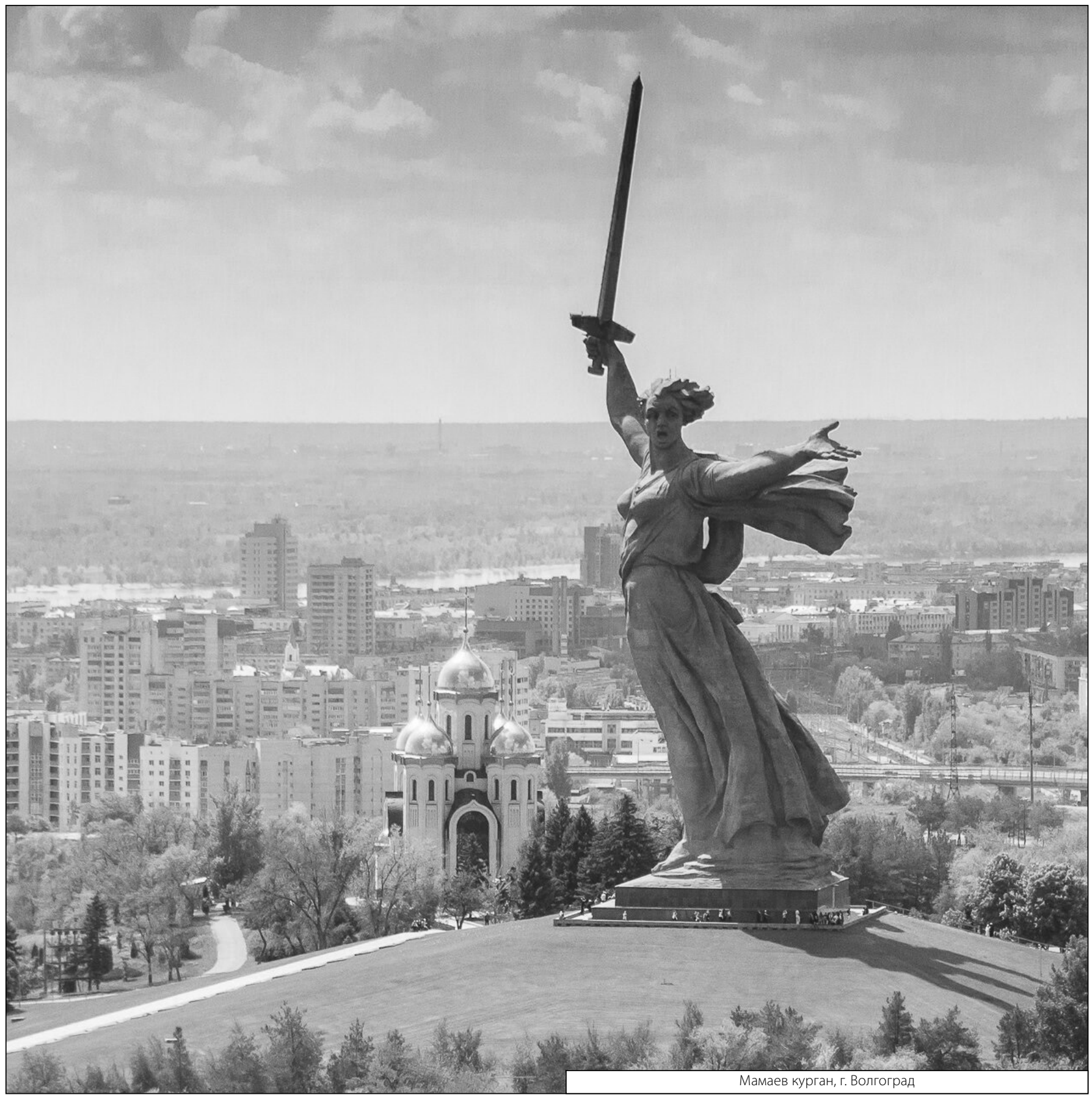

